\title{
Towards Fast and Accurate Neural Chinese Word Segmentation with Multi-Criteria Learning
}

\author{
Weipeng Huang* Xingyi Cheng* ${ }^{* \dagger}$ Kunlong Chen Taifeng Wang Wei Chu \\ Ant Group \\ \{weipeng.hwp, fanyin.cxy, \\ kunlong.ckl, taifeng.wang, weichu.cw\}eantgroup.com
}

\begin{abstract}
The ambiguous annotation criteria lead to divergence of Chinese Word Segmentation (CWS) datasets in various granularities. Multi-criteria Chinese word segmentation aims to capture various annotation criteria among datasets and leverage their common underlying knowledge. In this paper, we propose a domain adaptive segmenter to exploit diverse criteria of various datasets. Our model is based on Bidirectional Encoder Representations from Transformers (BERT), which is responsible for introducing open-domain knowledge. Private and shared projection layers are proposed to capture domain-specific knowledge and common knowledge, respectively. We also optimize computational efficiency via distillation, quantization, and compiler optimization. Experiments show that our segmenter outperforms the previous state of the art (SOTA) models on 10 CWS datasets with superior efficiency.
\end{abstract}

\section{Introduction}

Chinese Word Segmentation (CWS) is typically regarded as a low-level NLP task. Unlike English and French that uses the space token to separate the words, Chinese is a kind of polysynthetic languages where compounds are developed from indigenous morphemes (Jernudd and Shapiro, 2011; Gong et al., 2017). The ambiguous distinction between morphemes and compound words leads to the cognitive divergence of word concepts. Consequently, the labeled datasets seriously diverge due to the annotation inconsistency, resulting in multi-grained compounds. As shown in Table 1, given a sentence “刘国梁赢 得世界冠军” (Liu Guoliang wins the world championship), the two commonly used corpora, i.e., PKU's People's Daily (PKU) and Penn Chinese Treebank (CTB), use different segmentation criteria.

In practice, a segmenter usually provides multiple configures with different granularities to better serve various downstream tasks. Fine-grained criterion is able to reduce the vocabulary, thereby relieves the sparseness issue. On the other hand, coarse-grained words provide more specific meanings, which may benefit the domain-specific tasks.

In recent years, several multi-criteria learning methods for CWS have been proposed to explore the common knowledge of heterogeneous datasets. By utilizing the information across all corpora, multicriteria learning methods can boost the out-of-vocabulary (OOV) recalls as well as practical performance (Qiu et al., 2013; Chao et al., 2015; Chen et al., 2017). Despite its effectiveness, there still are three unresolved issues. (1) Even with multiple datasets, the data is still limited to provide adequate linguistic knowledge. (2) Learning from a dataset is likely to hurt the others as the segmentation follows inconsistent criteria. (3) The advanced models, e.g., Bi-LSTM-CRF (Ma et al., 2018), are computationally expensive. They are based on the recurrent neural networks (RNNs). Since RNNs are auto-regressive and the computation cannot be completed in parallel, their applications are usually limited due to the poor computational efficiency. In fact, the inference speed is heavily required for the CWS system as it serves

\footnotetext{
${ }^{*}$ Equal contribution.

${ }^{\dagger}$ Corresponding author.

This work is licensed under a Creative Commons Attribution 4.0 International Licence. Licence details: http:// creativecommons.org/licenses/by/4.0/.
} 


\begin{tabular}{c|c|c|c|c|c}
\hline Criteria & Liu & Guoliang & wins & \multicolumn{2}{|c}{ the world championship } \\
\hline CTB & \multicolumn{2}{|c|}{ 刘国梁 } & 赢得 & \multicolumn{2}{|c}{ 世界冠军 } \\
\hline PKU & 刘 & 国梁 & 赢得 & 世界 & 冠军 \\
\hline
\end{tabular}

Table 1: Diverse segmentation criteria.

as a fundamental module of NLP pipelines. For example, search engines generally can only afford to spend tens of milliseconds or even milliseconds in CWS.

To alleviate the limitations of existing methods, we propose a multi-criteria method for CWS. Recent studies (Yang et al., 2017; Ma et al., 2018; Wang et al., 2019) pointed out that exploiting external knowledge can improve the CWS accuracy. Based on this observation, we adopt BERT (Vaswani et al., 2017; Devlin et al., 2018) as the backbone to extract the open-domain knowledge. On the top of BERT, private projection layers and shared projection layers are used to capture domain-specific knowledge and common underlying knowledge respectively.

To make it more practicable, three techniques, i.e., knowledge distillation, numeric quantization and compiler optimization, are adopted to accelerate our segmenter. The BERTology analysis (Clark et al., 2019; Jawahar et al., 2019; Xu et al., 2020) indicated that the representations from different layers of BERT capture specific meanings. It is sufficient to use the representations from a middle layer for the CWS task (see section 4.5.2 for detailed analysis). To make the best use of BERT, the knowledge distillation method proposed by (Hinton et al., 2015) is utilized.

Simultaneously, we also adopt quantization and compiler optimization techniques to improve the scalability. Experiments show that our method not only significantly outperforms the best known results on 10 CWS datasets with better efficiency.

The contributions could be summarized as follows.

- BERT with a domain projection layer on the top is employed to capture heterogeneous segmentation criteria and common underlying knowledge. To our knowledge, it is the first time to utilize pretrained model in CWS.

- We visualize the BERT layers and attention scores to give an insight into linguistic information within CWS.

- Model acceleration techniques including distillation, quantization and compiler optimization, are adopted to improve the segmentation speed.

- Experimental results show that our model outperforms previous results on $10 \mathrm{CWS}$ corpora with different segmentation criteria.

\section{Model Description}

Current neural CWS models usually consist of three components: a character embedding layer, a feature extraction layer and a CRF tag inference layer. To equip our model with the ability of multi-criteria learning, we insert an extra domain projection layer before the inference layer, as shown in Figure 1. In this section, we describe the proposed model architecture and the objective function in detail.

\subsection{Feature Extraction Layer}

We employ BERT (Vaswani et al., 2017; Devlin et al., 2018) to extract feature for the input sequence. BERT is of critical importance for the word segmentation task. As shown in Figure 1, the characters are first mapped into embedding vectors and then fed into several transformer blocks. Compared with BiLSTM which processes the sequence step by step, the transformer learns features in parallel for all timesteps so that the decoding speed can be accelerated. However, the original BERT with 12 transformer layers is still too heavy to be applied in the real-world word segmentation application. To speed up both the fine-tuning and inference procedures, we make further optimization as discussed in Section 3. 


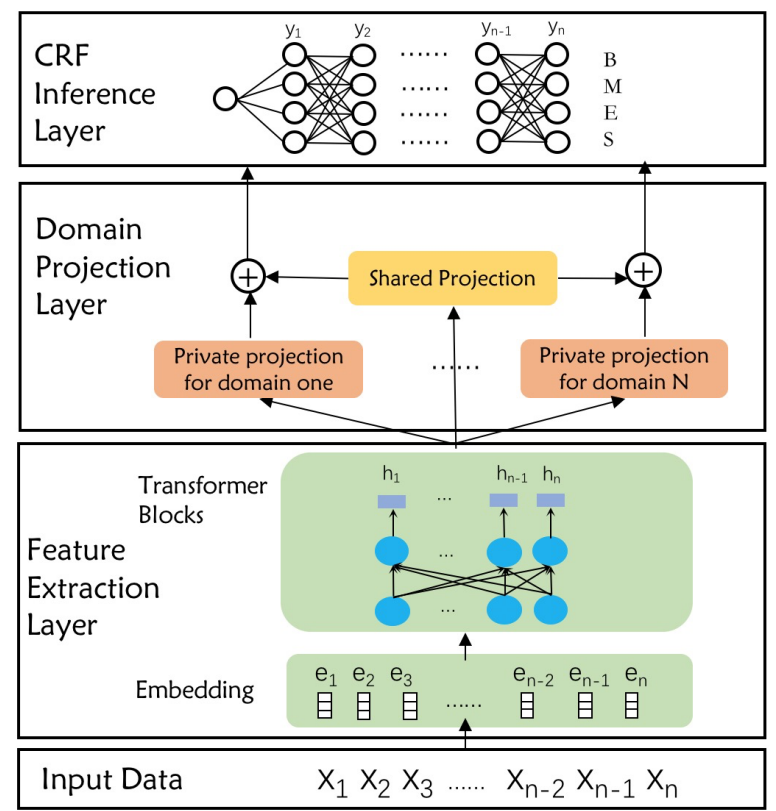

Figure 1: The architecture of the proposed model, stacked with a feature extraction layer, a domain projection layer and a CRF tag inference layer.

Given a sentence $X=\left\{x_{1}, x_{2}, \ldots, x_{n}\right\}$, we first map each character $x_{i}$ into embedding vector $\mathbf{e}_{i}$. The embedding vectors are then fed into BERT to get the feature representations:

$$
\mathbf{h}_{i}=\operatorname{BERT}\left(\mathbf{e}_{1}, \mathbf{e}_{2}, \ldots, \mathbf{e}_{n} ; \theta\right),
$$

where $\theta$ denotes all the parameters in BERT model.

\subsection{Domain Projection Layer}

As shown in Table 1, the same sentence can be segmented into different words according to different dataset criteria. If we simply combine the datasets to train a single model, the model will be confused by diverse criteria and thus hurt the performance. Traditional methods train an individual model for each dataset, which results in huge deployment costs in practice.

Inspired by previous works (Chen et al., 2017; Peng and Dredze, 2017), we propose a domain projection layer to enable our model to adapt the datasets with various criteria. The domain projection layer helps to capture heterogeneous segmentation criteria of each dataset. There are many variations of the projection layer; in this paper, we use a linear transformation layer, which is simple but effective for this task. As shown in Figure 1, we introduce an extra shared projection layer to learn common knowledge from datasets. The computation graph excludes domain-specific projections and reserves the shared projection to segment with standard criteria.

Formally, with the domain projection layer, we can obtain the domain-specific representation and shared representation:

$$
\begin{aligned}
\mathbf{h}_{\text {domain }} & =\mathbf{W}_{\text {domain }}^{T} \mathbf{h}+\mathbf{b}_{\text {domain }}, \\
\mathbf{h}_{\text {shared }} & =\mathbf{W}_{\text {shared }}^{T} \mathbf{h}+\mathbf{b}_{\text {shared }}
\end{aligned}
$$

where $\mathbf{W}_{\text {domain }}^{T} \in \mathbf{R}^{d_{h} \times d_{h}}, \mathbf{W}_{\text {shared }}^{T} \in \mathbf{R}^{d_{h} \times d_{h}}, \mathbf{b}_{\text {domain }} \in \mathbf{R}^{d_{h}}$ and $\mathbf{b}_{\text {shared }} \in \mathbf{R}^{d_{h}}$ are trainable parameters. $d_{h}$ is the dimension of $\mathbf{h}$.

\subsection{Inference Layer}

We treat the CWS task as a character-based sequence labeling problem. Each character in a sentence $X=\left\{x_{1}, x_{2}, \ldots, x_{n}\right\}$ is labelled as one of $\mathcal{L}=\{B, M, E, S\}$, indicating the begin, middle, end of a word, and a word with single character. As shown in Figure 1, the output of domain-specific projection 
and shared projection are concatenated, then fed into a first-order linear-chain conditional random fields (CRF) layer (Lafferty et al., 2001) to inference these tags.

Formally, the probability of a label sequence formalized as:

$$
p(Y \mid X)=\frac{\Psi(Y \mid X)}{\sum_{Y^{\prime} \in \mathcal{L}^{n}} \Psi\left(Y^{\prime} \mid X\right)},
$$

where $\Psi(Y \mid X)$ is potential function:

$$
\begin{aligned}
\Psi(Y \mid X) & =\prod_{i=2}^{n} \psi\left(X, i, y_{i-1}, y_{i}\right), \\
\psi\left(X, i, y_{i-1}, y_{i}\right) & =\exp \left(s(X, i)_{y_{i}}+\mathbf{b}_{y_{i-1} y_{i}}\right),
\end{aligned}
$$

where $y \in\{B, M, E, S\}$ is the tag label, $\mathbf{b} \in \mathbf{R}^{|\mathcal{L}| \times|\mathcal{L}|}$ is trainable parameter and $\mathbf{b}_{y_{i-1} y_{i}}$ means transition from tag $y_{i-1}$ to $y_{i}$. Score function $s(X, i)$ is output of the projection layer at $i_{t h}$ character, which assigns score for each label on tagging the $i_{t h}$ character:

$$
s(X, i)=\mathbf{W}_{s}^{T}\left[\mathbf{h}_{\text {domain }} ; \mathbf{h}_{\text {shared }}\right]+\mathbf{b}_{s},
$$

where $\left[\mathbf{h}_{\text {domain }} ; \mathbf{h}_{\text {shared }}\right]$ is the concatenation of domain-specific projection and shared projection, $\mathbf{W}_{s}^{T} \in$ $\mathbf{R}^{2 d_{h} \times|\mathcal{L}|}$ and $\mathbf{b}_{s} \in \mathbf{R}^{|\mathcal{L}|}$ are trainable parameters.

The inference can be achieved by maximizing the posterior probability:

$$
Y^{*}=\arg \max p(Y \mid X) \text {. }
$$

\subsection{Objective Function}

The parameters of the network are trained to maximize the conditional log-likelihood of true labels on the dataset. The objective function $\mathcal{J}_{\text {seg }}$ is computed as :

$$
\mathcal{J}_{\text {seg }}(\Theta)=\sum_{j} \log p\left(Y^{(j)} \mid X^{(j)} ; \Theta\right),
$$

where $\Theta$ denotes all the parameters in the model, $\left(X^{(j)}, Y^{(j)}\right)$ denotes the $j_{t h}$ sample in the datasets. The total loss for multi-criteria learning is the combination of loss in each datasets.

\section{Model Acceleration}

Neural CWS models improve the performance by increasing the model complexity, which however harms the decoding speed and limits their real-world application. To bridge this gap, we apply model acceleration techniques as follows.

\subsection{Distillation}

To balance computational cost and segmentation accuracy, we distill knowledge (Ba and Caruana, 2014; Hinton et al., 2015) from BERT into a smaller transformer network. And the supervised fine-tuning on the datasets are performed jointly. Recent analysis (Clark et al., 2019; Jawahar et al., 2019) show that the layers of BERT provide phrase-level information, the middle layers extract syntactic features and the top layers are capable of handling semantic features. CWS is essentially a syntactic chunking task and heavily relies on lexical and syntactic features. Therefore, we turn to use bottom-to-middle layers as the backbone to learn annotations and jointly distill the top layer of BERT. Specifically, the original Chinese BERT with 12 layers serve as a teacher, and a truncated (3 or 6 layers) BERT learns from the teacher as a student. The teacher network and student network differ in the feature extraction layer of our proposed model shown in Figure 1. 


\begin{tabular}{c|ccc|c}
\hline Datasets & Training set & Development set & Testing set & Average word length \\
\hline CNC & $5920 \mathrm{~K}$ & $657 \mathrm{~K}$ & $727 \mathrm{~K}$ & 1.52 \\
\hline AS & $4903 \mathrm{~K}$ & $546 \mathrm{~K}$ & $122 \mathrm{~K}$ & 1.51 \\
\hline MSR & $2132 \mathrm{~K}$ & $235 \mathrm{~K}$ & $106 \mathrm{~K}$ & 1.68 \\
\hline CITYU & $1309 \mathrm{~K}$ & $146 \mathrm{~K}$ & $40 \mathrm{~K}$ & 1.62 \\
\hline PKU & $994 \mathrm{~K}$ & $115 \mathrm{~K}$ & $104 \mathrm{~K}$ & 1.61 \\
\hline CTB6 & $641 \mathrm{~K}$ & $59 \mathrm{~K}$ & $81 \mathrm{~K}$ & 1.63 \\
\hline SXU & $476 \mathrm{~K}$ & $53 \mathrm{~K}$ & $113 \mathrm{~K}$ & 1.57 \\
\hline UD & $100 \mathrm{~K}$ & $11 \mathrm{~K}$ & $12 \mathrm{~K}$ & 1.49 \\
\hline ZX & $79 \mathrm{~K}$ & $9 \mathrm{~K}$ & $34 \mathrm{~K}$ & 1.42 \\
\hline WTB & $15 \mathrm{~K}$ & $1 \mathrm{~K}$ & $2 \mathrm{~K}$ & 1.53 \\
\hline
\end{tabular}

Table 2: Details of the ten datasets: the number of words in the training set, development set and testing set, the average word length (char/word) of each dataset.

\begin{tabular}{c|cccccccccc}
\hline & PKU & MSR & AS & CITYU & CTB6 & SXU & UD & CNC & WTB & ZX \\
\hline (Yang et al., 2017) & 96.3 & 97.5 & 95.7 & 96.9 & 96.2 & - & - & - & - & - \\
(Chen et al., 2017) & 94.3 & 96.0 & 94.6 & 95.6 & 96.2 & 96.0 & - & - & - & - \\
(Xu and Sun, 2017) & 96.1 & 96.3 & - & - & 95.8 & - & - & - & - & - \\
(Ma et al., 2018) & 96.1 & 98.1 & 96.2 & 97.2 & 96.7 & - & 96.9 & - & - & - \\
(Gong et al., 2018) & 96.2 & 97.8 & 95.2 & 96.2 & 97.3 & 97.2 & - & - & - & - \\
(Zhou et al., 2019) & 96.2 & 97.0 & 96.9 & 97.1 & 95.2 & - & - & - & - & - \\
(He, 2019) & 96.0 & 97.2 & 95.4 & 96.1 & 96.7 & 96.4 & 94.4 & 97.0 & 90.4 & 95.7 \\
\hline Ours (Student-3 layer) & 96.7 & 97.9 & 96.8 & 97.6 & 97.5 & 97.3 & 97.4 & 97.1 & 93.1 & 97.0 \\
Ours (Student-3 layer+FP16) & 96.6 & 98.0 & 96.6 & 97.5 & 97.4 & 97.3 & 97.3 & 97.1 & 92.7 & 96.8 \\
Ours (Student-6 layer) & 97.2 & 98.3 & 97.0 & 97.7 & 97.7 & $\mathbf{9 7 . 5}$ & $\mathbf{9 7 . 8}$ & 97.2 & 93.0 & $\mathbf{9 7 . 1}$ \\
Ours (Student-6 layer+FP16) & 97.0 & 98.2 & 96.8 & $\mathbf{9 7 . 8}$ & 97.7 & 97.4 & 97.7 & 97.1 & 93.1 & 96.8 \\
Ours (Teacher-12 layer) & $\mathbf{9 7 . 3}$ & $\mathbf{9 8 . 5}$ & $\mathbf{9 7 . 0}$ & $\mathbf{9 7 . 8}$ & $\mathbf{9 7 . 8}$ & $\mathbf{9 7 . 5}$ & $\mathbf{9 7 . 8}$ & $\mathbf{9 7 . 3}$ & $\mathbf{9 3 . 2}$ & $\mathbf{9 7 . 1}$ \\
Ours (Teacher-12 layer+FP16) & 97.2 & 98.3 & 96.9 & $\mathbf{9 7 . 8}$ & 97.7 & 97.3 & 97.7 & 97.2 & 93.1 & 96.9 \\
\hline
\end{tabular}

Table 3: Comparison among the state-of-the-art performance on different datasets (F1-score, \%).

To distill the original BERT, we add a logits-regression objective by minimizing the square loss between the normalized logits from the teacher model and the logits from the student model. The distillation loss is formulated as:

$$
\mathcal{J}_{\text {dis }}\left(\Theta_{s}, \Theta_{t}\right)=\frac{1}{2 T} \sum_{j=0}^{M} \sum_{i=0}^{N}\left(\frac{\mathbf{h}_{s}^{(j, i)}}{\left\|\mathbf{h}_{s}^{(j, i)}\right\|_{2}}-\frac{\mathbf{h}_{t}^{(j, i)}}{\left\|\mathbf{h}_{t}^{(j, i)}\right\|_{2}}\right)^{2},
$$

where $\Theta_{s}, \Theta_{t}$ denote parameters in the student network and teacher network, $M$ denotes the number of samples in the datasets, $N$ denotes the sequence length, $\mathbf{h}_{s}, \mathbf{h}_{t}$ denote the logits extracted from student network and teacher network respectively. During the distillation process, the parameters $\Theta_{t}$ are frozen.

Combining the segmentation loss and the distillation loss, the overall loss is:

$$
\mathcal{J}\left(\Theta_{s}, \Theta_{t}\right)=\mathcal{J}_{\text {seg }}\left(\Theta_{s}\right)+\alpha \mathcal{J}_{\text {dis }}\left(\Theta_{s}, \Theta_{t}\right),
$$

where $\alpha$ is a hyper-parameter to trade off these two loss function.

\subsection{Quantization}

Quantization methods also have been investigated for network acceleration. These approaches are mainly categorized into two groups: scalar and vector quantization (Gong et al., 2014), fixed-point quantization (Gupta et al., 2015). Traditional neural networks implementation use 32-bit single-precision floatingpoint for both weights and activation, resulting in a cost of the substantial increase in computation and model storage resources. Therefore, we conduct fixed-point quantization to leverage NVIDIA's Volta architectural features. Fixed-point quantization was proposed to alleviate these complexities.

Specifically, in our model, half-precision (FP16) is applied on kernels of multi-head attention layers and feedforward layers, while rest parameters like embedding and normalization parameters use full precision (FP32). Gradients in fine-tuning procedures also use full precision. The quantization method not only accelerates the computation but also reduce the model size. 


\begin{tabular}{c|ccc}
\hline & Precision & Recall & F1-Score \\
\hline Teacher-BERT (12 layer) & 97.2 & 97.0 & 97.1 \\
Student-Transformer (6 layer) & 97.1 & 97.0 & 97.0 \\
Student-Transformer (3 layer)) & 96.8 & 96.9 & 96.8 \\
Student-Transformer (1 layer) & 95.9 & 96.1 & 96.0 \\
\hline
\end{tabular}

Table 4: Average Precision, Recall, F1-score on 10 datasets for the teacher network and the student network.

\begin{tabular}{c|c|c|c|c|c|c|c|c|c|c|c}
\hline & AS & CITYU & CNC & CTB6 & MSR & PKU & SXU & UD & WTB & ZX & All Datasets \\
\hline AS & 0.0 & 13.9 & 14.8 & 8.4 & 7.1 & 6.4 & 4.5 & 2.3 & 0.4 & 0.9 & 30.3 \\
\hline CITYU & 33.5 & 0.0 & 31.4 & 14.0 & 20.9 & 17.5 & 9.8 & 4.0 & 0.8 & 2.4 & 50.5 \\
\hline CNC & 14.8 & 6.2 & 0.0 & 4.2 & 7.7 & 6.0 & 2.7 & 1.7 & 0.2 & 0.6 & 25.1 \\
\hline CTB6 & 47.3 & 31.2 & 40.5 & 0.0 & 28.0 & 24.6 & 15.4 & 7.1 & 1.2 & 3.1 & 63.9 \\
\hline MSR & 18.4 & 10.5 & 27.3 & 8.0 & 0.0 & 10.7 & 5.9 & 2.3 & 0.1 & 1.3 & 36.0 \\
\hline PKU & 39.6 & 31.7 & 50.1 & 25.3 & 35.2 & 0.0 & 15.7 & 8.6 & 0.7 & 3.9 & 67.3 \\
\hline SXU & 49.8 & 39.7 & 56.2 & 29.3 & 41.8 & 36.6 & 0.0 & 10.9 & 1.7 & 4.9 & 73.2 \\
\hline UD & 55.3 & 43.2 & 57.5 & 37.6 & 45.1 & 37.2 & 30.2 & 0.0 & 3.8 & 6.2 & 70.2 \\
\hline WTB & 76.5 & 71.0 & 79.2 & 64.3 & 72.5 & 69.8 & 65.1 & 41.6 & 0.0 & 22.0 & 85.1 \\
\hline ZX & 71.4 & 49.8 & 71.8 & 43.0 & 53.1 & 44.2 & 35.3 & 20.1 & 6.9 & 0.0 & 81.1 \\
\hline
\end{tabular}

Table 5: Each row indicates the rate of OOV words ( \% ) in a dataset appear in other datasets.

\subsection{Compiler Optimization}

XLA (Accelerated Linear Algebra) is a domain-specific compiler for linear algebra that accelerates TensorFlow models by optimizing one's computations. It provides an alternative mode of running TensorFlow models: it compiles the TensorFlow graph into a sequence of computation kernels generated specifically for the given model. Because these kernels are unique to the model, they can exploit modelspecific information for optimization. For example, operations like addition, multiplication and reduction can be fused into a single GPU kernel.

By introducing XLA into our model, graphs are compiled into machine instructions, and low-level ops are fused to improve the execution speed. For example, batch matmul is always followed by a transpose operation in the transformer computation graph. By fusing these two operations, the intermediate product does not need to write back to memory, thus reducing the redundant memory access time and kernel launch overhead.

\section{Experiments}

\subsection{Experimental Settings}

All experiments are implemented on the hardware with Intel(R) Xeon(R) CPU E5-2682 v4 @ 2.50GHz and NVIDIA Tesla V100.

Datasets. We evaluate our model on ten standard Chinese word segmentation datasets: MSR,PKU,AS,CITYU from SIGHAN 2005 bake-off task (Emerson, 2005). SXU from SIGHAN 2008 bake-off task (MOE, 2008). Chinese Penn Treebank 6.0 (CTB6) from (Xue et al., 2005). Chinese Universal Treebank (UD) from the Conll2017 shared task (Zeman and Popel, 2017). WTB (Wang and Yang, 2014), ZX (Zhang and Meishan, 2014) and CNC corpus. For each of the SIGHAN 2005 and 2008

\begin{tabular}{c|c|cccccccccc|c}
\hline & & PKU & MSR & AS & CITYU & CTB6 & SXU & UD & CNC & WTB & ZX & Avg \\
\hline F1 & single-criteria & 94.7 & 95.3 & 95.2 & 95.7 & 95.4 & 94.4 & 94.6 & 96.3 & 89.9 & 94.4 & 94.5 \\
Score & multi-criteria & $\mathbf{9 6 . 7}$ & $\mathbf{9 7 . 9}$ & $\mathbf{9 6 . 7}$ & $\mathbf{9 7 . 6}$ & $\mathbf{9 7 . 5}$ & $\mathbf{9 7 . 3}$ & $\mathbf{9 7 . 4}$ & $\mathbf{9 7 . 1}$ & $\mathbf{9 3 . 1}$ & $\mathbf{9 7 . 0}$ & $\mathbf{9 6 . 8}$ \\
\hline OOV & single-criteria & 74.8 & 78.0 & $\mathbf{7 8 . 3}$ & 83.7 & 62.8 & 80.1 & 73.6 & 64.2 & 73.9 & 74.8 & 74.2 \\
Recall & multi-criteria & $\mathbf{8 1 . 6}$ & $\mathbf{8 4 . 0}$ & 77.3 & $\mathbf{9 0 . 1}$ & $\mathbf{8 9 . 4}$ & $\mathbf{8 5 . 7}$ & $\mathbf{9 1 . 6}$ & $\mathbf{6 5 . 0}$ & $\mathbf{8 2 . 9}$ & $\mathbf{8 9 . 1}$ & $\mathbf{8 3 . 6}$ \\
\hline
\end{tabular}

Table 6: OOV recall(\%), F1 Score (\%) achieved with multi-criteria learning and single-criteria learning. The number of transformer layer is set to 3 for both single-criteria and multi-criteria learning. 
dataset, we randomly select $10 \%$ training data as the development set. For other datasets, we use official data split. Table 2 shows the details of the ten datasets. We can notice that the average word length (char per word) of these datasets range from 1.42 to 1.68 , which reflects the diverse segmentation granularities and data distribution of these datasets.

Preprocessing. AS and CITYU are mapped from traditional Chinese to simplified Chinese before segmentation. Continuous English characters and digits in the datasets are respectively replaced with a unique token. Full-width tokens are converted to half-width to handle the mismatch between training and testing set.

Hyperparameters. The number of domain projection layer is 1 , the max sequence length is set to 128. During fine-tuning, we use Adam with the learning rate of 2e-5, L2 weight decay of 0.01 , dropout probability of 0.1 . For the trade-off hyperparameter $\alpha$, we had tried several value and empirically fixed it to 0.15 in the following experiment. Parameters in the feature extraction layer of teacher network and student network are initialized with pre-trained BERT ${ }^{1}$, and all other parameters are initialized with Xavier uniform initializer.

Evaluation Metrics. The goal of Chinese word segmentation is to precisely cut the input sentence into separate words. Therefore, to reach a balance of the precision $\left(P=\frac{\# \text { word }_{\text {gold } \cap \text { sys }}}{\# \text { ord }_{\text {sys }}}\right)$ and recall $\left(R=\frac{\# \text { word }_{\text {gold } n \text { sys }}}{\# \text { word }}\right)$, we use the $\mathrm{F} 1$ score $\left(R=\frac{2 P R}{P+R}\right)$.

\subsection{Main Results}

We distill the teacher network with the truncated BERT that compared with using the original 12 layers BERT. The average F1-score on 10 datasets using 3 layers drops slightly from $97.1 \%$ to $96.8 \%$ as shown in Table 4 . We suggest a student network with 3 transformer layers is a good choice to balance computational cost and segmentation accuracy.

Performance of our model and recent neural CWS models are shown in Table 3. Our model outperform prior works on 10 datasets, with $13.5 \%, 10.5 \%, 15.8 \%, 17.9 \%, 14.8 \%, 10.7 \%, 32.2 \%, 10.0 \%$, $29.1 \%, 32.5 \%$ error reductions on PKU, MSR, AS, CITYU, CTB6, SXU, UD, CNC, WTB, ZX datasets respectively. By further applying half-precision (FP16), the accuracy reduction is minor and the model still outperforms previous SOTA results on 10 datasets. The F1 score did not change when applying compiler optimization since it had no effect on the result of the predictions.

\subsection{Effect of Domain Projection Layer}

Previous work (Huang and Zhao, 2007; Ma et al., 2018) pointed out that OOV is a major error and exploring further sources of knowledge is essential to solving this problem. From a certain point of view, datasets are complementary to each other since OOV in a dataset may appear in other datasets. We make some analysis and the statistics are shown in Table 5. Take the dataset AS for example, $13.9 \%$ of the OOV words appear in dataset CITYU, and 30.3\% of the OOV words appear in all other datasets.

To utilize knowledge from each other to improve the OOV recall, our model performs multi-criteria learning with the domain projection layer. To evaluate this, we train the proposed model respectively on each dataset, i.e., single-criteria learning. In single-criteria learning setting, the shared projection layer is excluded and only the private projection layer is preserved for each dataset. The number of student transformer layers is set to 3 for both single-criteria and multi-criteria learning. Table 6 shows that comparing with single-criteria learning, multi-criteria learning significantly improves the F1 score on all datasets, with $2.3 \%$ improvement on average. It also improves the OOV recall on 9/10 datasets, with $9.4 \%$ improvement on average.

\subsection{Scalability}

Decoding speed is essential in practice since word segmentation is fundamental for many downstream NLP tasks. Previous neural CWS models (Ma et al., 2018; Chen et al., 2017; Gong et al., 2018; Zhou et al., 2019) use Bi-LSTM with concatenated embedding size of 100,100,128,100 respectively. However,

\footnotetext{
${ }^{1}$ https://github.com/google-research/bert
} 


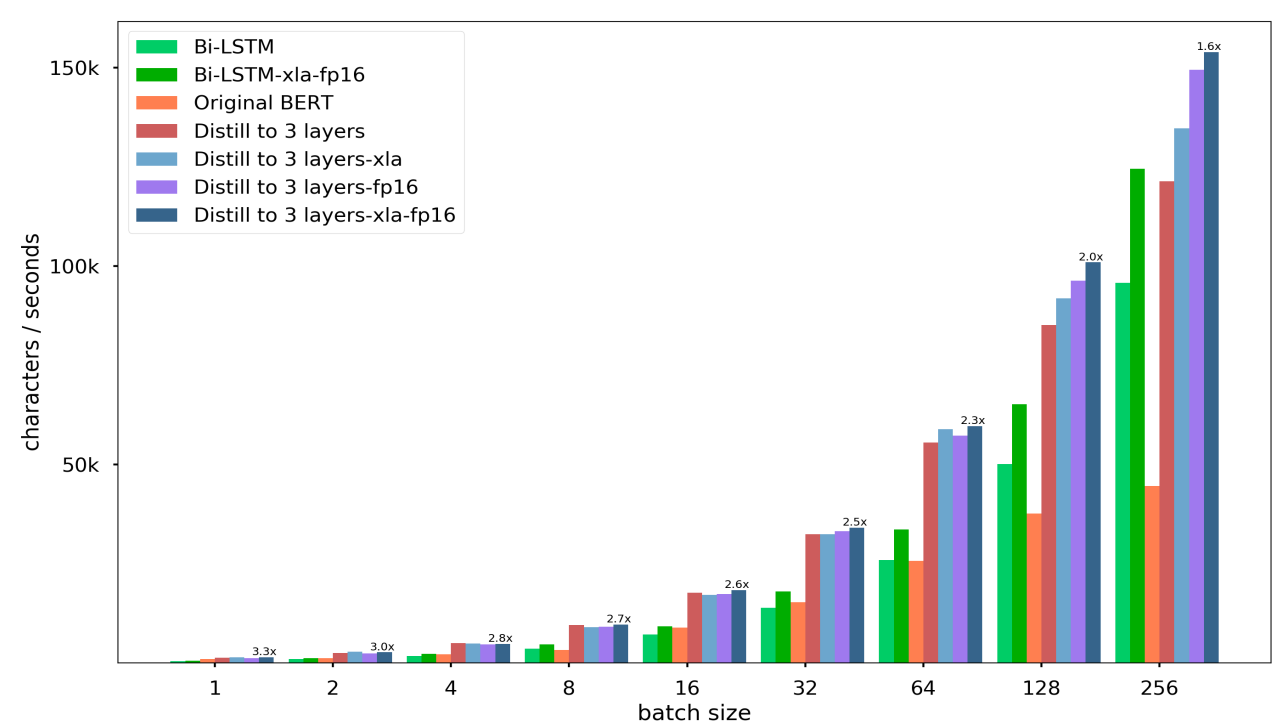

Figure 2: Decoding speed w.r.t batch size. The sequence length is 64 .

they did not report the decoding speed. To make a fair comparison, we set the Bi-LSTM embedding size and hidden size to 100 , one hidden layer with CRF on the top.

Figure 2 shows the decoding speed with regards to batch size. Our model employed original Chinese BERT with 12 transformer layers is slower than Bi-LSTM. However, the speed can be increased by optimizations, including distillation, weights quantization, and compiler optimization. Combining all of these three techniques, our models outperform Bi-LSTM with $1.6 \times-3.3 \times$ acceleration with different batch size. We try to adapt Bi-LSTM with the same computational optimization as in BERT. The result shows that Bi-LSTM achieved about $30 \%$ acceleration by optimization. And our method still outperforms the optimized Bi-LSTM, i.e. Bi-LSTM-xla-fp16, with $1.3 \times-2.5 \times$ acceleration. Furthermore, our model is more scalable compared with the Bi-LSTM that are limited in their capability to process tasks involving very long sequences. By observing the sequence length distribution, we can search an appropriate layer number to balance F1-score and decoding speed.

\subsection{Visualization}

\subsubsection{Self Attention Score Visualization}

In the self-attention layers of the transformer, the attention score of each character is calculated with the rest characters. By visualizing the attention score, we can intuitively see what each character pay attention to. Specifically, we choose the sentences with a length larger than 50 in all the ten datasets, feed these sentences into the model and average the attention scores at each index. The attention score is shown in Figure 3(a)(b)(c). We can notice that characters around the current character gain larger weights than those far away. The result indicates that word segmentation depends more on phrase-level information and long term dependencies are relatively unimportant. It intuitively proves that it is not necessary to keep long term memory of the sequence for CWS.

\subsubsection{Layer Attention Score Visualization}

BERT has achieved great success in many NLU tasks by pre-training a stack of 12 transformer layers to learn abundant knowledge. Intuitively, top layers capture high-level semantic features while bottom layers learn low-level features like grammar. As for chinese word segmentation task, high-level semantic features may have a small impact so that we make further investigation to find the minimal number of transformer layers. We freeze weights of each layer in the pre-trained BERT and conduct layer attention fine-tuning on word segmentation datasets. As shown in Figure 3(d), the attention score gradually decrease in top layers from 7 to 12 , and the third layer gains the highest attention score. The results show that the model with three layers contains most information for word segmentation. Recent BERTol- 


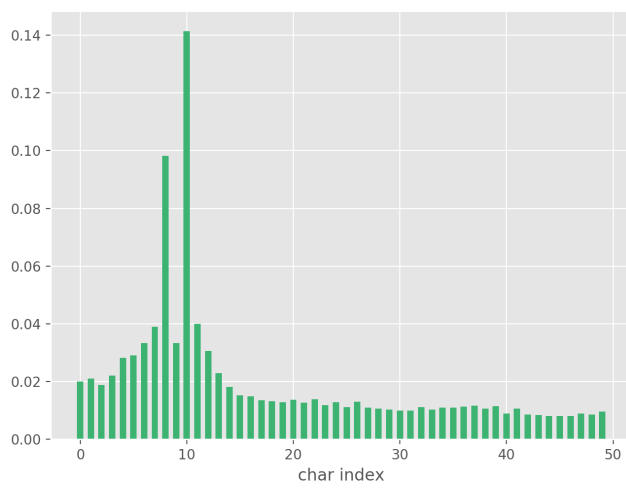

(a) Current char index 10

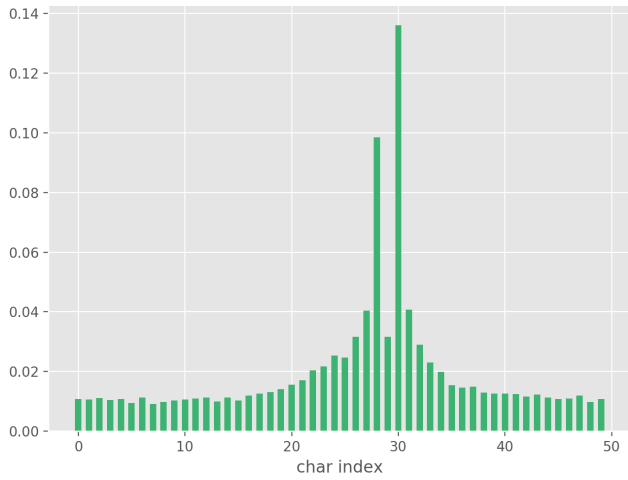

(c) Current char index 30

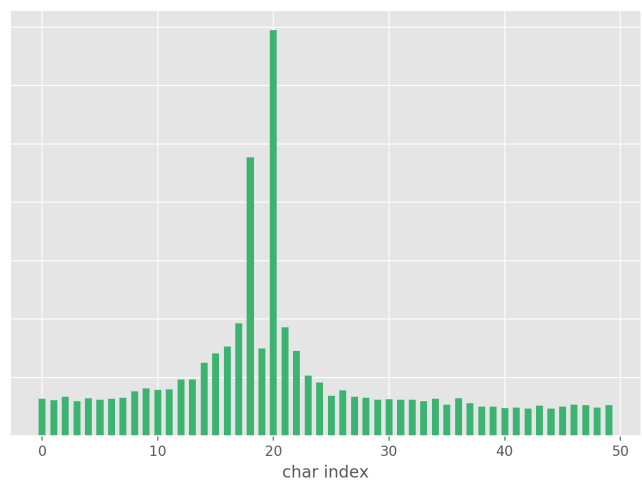

(b) Current char index 20

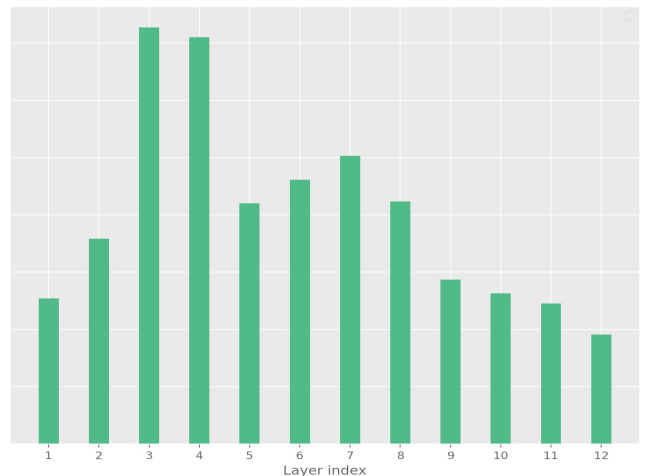

(d) Layer attention score

Figure 3: Distribution of self-attention score at certain char index $(10,20,30)$ and layer attention score.

ogy (Jawahar et al., 2019; Liu et al., 2019; Tenney et al., 2019; Hewitt and Manning, 2019; Goldberg, 2019) analysis aimed to understand the inner working of BERT from the perspective of linguistics. These works came into a similar conclusion that the basic syntactic information of chunking appears in lower or middle layers, which is consistent with our analysis. The higher layers of BERT are the most semantics or tasks-specific. These analyses also reflect that Chinese word segmentation is a task of more syntactic but less partial semantic knowledge in linguistics.

\section{Related Work}

Recently, multi-criteria learning of neural CWS has drawn great attention of scholars. (Qiu et al., 2013) adopted the stack-based model to take advantage of annotated data from multiple sources. (Chao et al., 2015) utilize multiple corpora using coupled sequence labeling model to learn and infer two heterogeneous annotations directly. (Gong et al., 2018) proposed Switch-LSTM to improve the performance of every single criterion by exploiting the underlying shared sub-criteria across multiple heterogeneous criteria. (Chen et al., 2017) have proposed a multi-criteria learning framework for CWS. They proposed three shared-private models to integrate multiple segmentation criteria. An adversarial strategy is used to force the shared layer to learn criteria-invariant features. All these works utilize heterogeneous annotation data and show that they can indeed help improve each other.

Model compression and acceleration in deep networks are vital in practice, which makes it possible to deploy deep models on mobile, embedded, and IoT devices. Techniques like parameter pruning, lowrank factorization, quantization and knowledge distillation had been widely used in visual tasks (Hinton et al., 2015; Ba and Caruana, 2014; Gupta et al., 2015; Gong et al., 2014). However, model compression and acceleration are rarely investigated in NLP tasks, especially neural CWS task. To the best of our knowledge, we are the first to compress the neural CWS model to accelerate the segmentation speed, by three model acceleration techniques, knowledge distillation, quantization and compiler optimization. We emphasize the segmentation speed is very important in industrial application, e.g., search engine. 


\section{Conclusion}

In this paper, we propose an effective Chinese Word Segmentation method that employs BERT and adds a domain projection layer on the top with multi-criteria learning. They both serve to capture heterogeneous segmentation criteria and common underlying knowledge. And we visualize the attention score to illustrate linguistic within CWS. To be practicability, acceleration techniques are applied to improve the word segmentation speed. It consists of knowledge distillation, quantization and compiler optimization. Experiments show that our proposed model achieves higher performance on the word segmentation accuracy and faster prediction speed than the state-of-the-art methods.

\section{References}

Jimmy Ba and Rich Caruana. 2014. Do deep nets really need to be deep? In Advances in neural information processing systems, pages 2654-2662.

Jiayuan Chao, Zhenghua Li, Wenliang Chen, and Min Zhang. 2015. Exploiting heterogeneous annotations for weibo word segmentation and pos tagging. In Natural Language Processing and Chinese Computing, pages 495-506. Springer.

X. Chen, Z. Shi, X. Qiu, and X Huang. 2017. Adversarial multi-criteria learning for chinese word segmentation. In Proceedings of the 55th Annual Meeting of the Association for Computational Linguistics, volume 1, pages 1193-1203.

Kevin Clark, Urvashi Khandelwal, Omer Levy, and Christopher D Manning. 2019. What does bert look at? an analysis of bert's attention. arXiv preprint arXiv:1906.04341.

Jacob Devlin, Ming-Wei Chang, Kenton Lee, and Kristina Toutanova. 2018. Bert: Pre-training of deep bidirectional transformers for language understanding. arXiv preprint arXiv:1810.04805.

Thomas Emerson. 2005. The second international chinese word segmentation bakeoff. In Proceedings of the fourth SIGHAN workshop on Chinese language Processing.

Yoav Goldberg. 2019. Assessing bert's syntactic abilities. arXiv preprint arXiv:1901.05287.

Yunchao Gong, Liu Liu, Ming Yang, and Lubomir Bourdev. 2014. Compressing deep convolutional networks using vector quantization. arXiv preprint arXiv:1412.6115.

Chen Gong, Zhenghua Li, Min Zhang, and Xinzhou Jiang. 2017. Multi-grained chinese word segmentation. In Proceedings of the 2017 Conference on Empirical Methods in Natural Language Processing, pages 692-703.

Jingjing Gong, Xinchi Chen, Tao Gui, and Xipeng Qiu. 2018. Switch-lstms for multi-criteria chinese word segmentation. arXiv preprint arXiv:1812.08033.

Suyog Gupta, Ankur Agrawal, Kailash Gopalakrishnan, and Pritish Narayanan. 2015. Deep learning with limited numerical precision. In International Conference on Machine Learning, pages 1737-1746.

Han He. 2019. Effective neural solution for multi-criteria word segmentation. pages 133-142. Smart Intelligent Computing and Applications. Springer.

John Hewitt and Christopher D Manning. 2019. A structural probe for finding syntax in word representations. In Proceedings of the 2019 Conference of the North American Chapter of the Association for Computational Linguistics: Human Language Technologies, Volume 1 (Long and Short Papers), pages 4129-4138.

Geoffrey Hinton, Oriol Vinyals, and Jeff Dean. 2015. Distilling the knowledge in a neural network. arXiv preprint arXiv:1503.02531.

Changning Huang and Hai Zhao. 2007. Chinese word segmentation: A decade review. Journal of Chinese Information Processing, 21(3):8-20.

Ganesh Jawahar, Benoît Sagot, Djamé Seddah, Samuel Unicomb, Gerardo Iñiguez, Márton Karsai, Yannick Léo, Márton Karsai, Carlos Sarraute, Éric Fleury, et al. 2019. What does bert learn about the structure of language? In 57th Annual Meeting of the Association for Computational Linguistics (ACL), Florence, Italy.

Björn H Jernudd and Michael J Shapiro. 2011. The politics of language purism, volume 54. Walter de Gruyter. 
J Lafferty, A McCallum, and F C N Pereira. 2001. Conditional random fields: Probabilistic models for segmenting and labeling sequence data.

Nelson F Liu, Matt Gardner, Yonatan Belinkov, Matthew Peters, and Noah A Smith. 2019. Linguistic knowledge and transferability of contextual representations. arXiv preprint arXiv:1903.08855.

Ji Ma, Kuzman Ganchev, and David Weiss. 2018. State-of-the-art chinese word segmentation with bi-lstms. In Proceedings of the 2018 Conference on Empirical Methods in Natural Language Processing.

PRC MOE. 2008. The fourth international chinese language processing bakeoff: Chinese word segmentation, named entity recognition and chinese pos tagging. In Proceedings of the sixth SIGHAN workshop on Chinese language processing.

N Peng and M Dredze. 2017. Multi-task domain adaptation for sequence tagging. In Proceedings of the 2nd Workshop on Representation Learning for NLP, pages 91-100.

Xipeng Qiu, Jiayi Zhao, and Xuanjing Huang. 2013. Joint chinese word segmentation and pos tagging on heterogeneous annotated corpora with multiple task learning. In Proceedings of the 2013 Conference on Empirical Methods in Natural Language Processing, pages 658-668.

Ian Tenney, Dipanjan Das, and Ellie Pavlick. 2019. Bert rediscovers the classical nlp pipeline. arXiv preprint arXiv:1905.05950.

Ashish Vaswani, Noam Shazeer, Niki Parmar, Jakob Uszkoreit, Llion Jones, Aidan N Gomez, Łukasz Kaiser, and Illia Polosukhin. 2017. Attention is all you need. In Advances in Neural Information Processing Systems, pages 5998-6008.

Wang and William Yang. 2014. Dependency parsing for weibo: An efficient probabilistic logic programming approach. In Proceedings of the 2014 conference on empirical methods in natural language processing $(E M N L P)$, pages $1152-1158$.

Xiaobin Wang, Deng Cai, Linlin Li, Guangwei Xu, Hai Zhao, and Luo Si. 2019. Unsupervised learning helps supervised neural word segmentation.

J Xu and X Sun. 2017. Dependency-based gated recursive neural network for chinese word segmentation. In The 54th Annual Meeting of the Association for Computational Linguistics, pages 1193-1203.

Weidi Xu, Xingyi Cheng, Kunlong Chen, and Taifeng Wang. 2020. Symmetric regularization based bert for pairwise semantic reasoning. In Proceedings of the 43rd International ACM SIGIR Conference on Research and Development in Information Retrieval, pages 1901-1904.

Naiwen Xue, Fei Xia, Fu-Dong Chiou, and Marta Palmer. 2005. The penn chinese treebank: Phrase structure annotation of a large corpus. Natural language engineering, 11(02):207ð̌C238.

J Yang, Y Zhang, and F Dong. 2017. Neural word segmentation with rich pretraining. In Proceedings of the 55th Annual Meeting of the Association for Computational Linguistics, pages 839-849.

Zeman and Martin Popel. 2017. Conll 2017 shared task: Multilingual parsing from raw text to universal dependencies. In Proceedings of the CoNLL 2017 Shared Task: Multilingual Parsing from Raw Text to Universal Dependencies, pages 1-19. Association for Computational Linguistics.

Zhang and Meishan. 2014. Type-supervised domain adaptation for joint segmentation and pos-tagging. In Proceedings of the 14th Conference of the European Chapter of the Association for Computational Linguistics, pages 588-597.

Jianing Zhou, Jingkang Wang, and Gongshen Liu. 2019. Multiple character embeddings for chinese word segmentation. In Proceedings of the 57th Conference of the Association for Computational Linguistics: Student Research Workshop, pages 210-216. 